\title{
Under-5s Swimming as a Site for Capital Building: Supporting and Enhancing Transitions
}

\begin{abstract}
Current government policy emphasises the importance of the preschool years engaging socially disadvantaged groups in formal learning. While significant resources are allocated for new centres that enable wider access for children to preschool education, it is argued in this paper that under-5s Swimming is a largely unrecognised and under-analysed activity that builds capital that enhances transition from home to school. In other words, high-quality learn-toswim classes may enhance benefits young children gain from their preschool experience. These claims are explored by drawing on the theoretical underpinnings of a large project concerned with the potential of the under- 5 swimming environment to enhance young children's learning. The paper is framed using and extending Bourdieu's notion of capital.
\end{abstract}

Across Australia there has been a coordinated movement by governments to create a system of childcare that addresses the needs of the nation. The goal is to enable every Australian child to have access to Early Childhood Education (ECE) by 2013. The Government has committed \$970million (Department for Education Employment and Workplace Relations, 2010) to facilitate this provision. This is a significant level of commitment to, and acknowledgement of, the importance of early childhood education to Australian society and to a wider region. It also acknowledges that in the past many children have been denied access to the benefits of early years education (Magnuson, Meyers, Ruhm, \& Waldfogel, 2004). While there is an explicit recognition that formal ECE learning contexts prepare children for the transition into schooling through particular programs (Carolyn. WebsterStratton \& Reid, 2004 ; Carolyn. Webster-Stratton, Reid, \& Stoolmiller, 2008) consideration of the breadth of potential areas for school preparation is necessary. Much of the focus is in the social development of the child. This paper draws on research from another context early-years swimming - to argue the potential in other contexts to help build the rich repertoire of learning that can help young children to transition into school. Swim-school environments and methods of instruction offer different opportunities that may further complement and enhance transitions into school.

This paper represents the theoretical framing of a large, three-year study on the effects of early-years swimming. The study is the first international study on the possible benefits for young children participating in swimming. As a nation where swimming is an integral part of the national identity, and death by accidental drowning is the major cause of deaths in under-fives, many young Australians participate in swimming. The swim industry estimates that approximately $10 \%$ of under-fives participate in swimming. Swimming is the most popular sport undertaken by pre-teens and, thus, it is important to understand the possible benefits of participating in the sport, particularly for preschool children. As swimming lessons are commenced at very young age, it is likely that it is the first encounter for many children with the instructional discourses they will encounter in formal schooling. As such, it offers a site for exploring the possibilities for adding to young Australians in ways that extend beyond swimming per se. 


\section{Swimming as a Capital Building Enterprise}

Rather than explain the changes in children's growth in terms of 'development', this paper draws on the notion of 'capital adding'. The position is shaped by the sociological lens of Bourdieu (Bourdieu, 1983). By framing the changes as forms of capital that can be enhanced through participation in early-years education, the various forms of capital become important considerations in the ways in which ECE may contribute to the success of learners. This paper considers the early-years swimming context (under $5 \mathrm{~s}$ ) as a site where forms of capital may be added to young learners in ways that may be different from more formal settings. In particular, the project is exploring the ways in which linguistic, social, intellectual, and physical capital potentially can be added to young children and support their transition to school. The transition to school requires a set of skills and dispositions that I refer to as school capital.

Unlike other areas of physical education where activities are constrained by the physical development of the child, early-years swimming can commence as soon as the baby is born. For example, in the approach used by used by Laurie Lawrence (http://www.youtube.com/watch?v=9W0A7rUAmv4), he advocates babies commence water familiarisation (which he argues is part of the swimming process) as part of the postnatal activities undertaken in the early weeks of life. The Federal government has sponsored Laurie Lawrence in the production of a swim DVD that is given to all new mothers as part of their new baby package. A little later, swimming can commence before the child is able to hold their head unaided. Predominantly swim schools commence formal lessons at 6 months but as noted earlier in this paper, some may start earlier. The parent is usually in the water with the child until it is 2-3 years old (depending on the swim school) and will support the child during lessons over this period of time. Early years swimming in these situations becomes an extension of child rearing and has the potential over time to be the basis of a form of capital building. This capital can be added through the use of language, the giving of directions in a three-dimensional space, the rewarding of behaviours, and the encouragement of children to enjoy and explore movement. The richness of the swimming environment extends the possibilities of the bath in the home environment so that the child perceives movement in a new space and gains a new world for experiencing aspects of physical space - e.g. up/down, under/over, and so on (Merleau-Ponty, 1961) that are not available in the day-to-day experiences. Many activities (such as easy tumbling, or moving through space) can be undertaken considerably earlier in the water environment than in the non-water environment, as the body is supported in water in ways that are not possible on land. This means that swimming can commence much earlier than other physical sports ${ }^{1}$. It also means that it is likely to be the first activity undertaken by children in a formal learning context where routines of an instructional discourse are present.

To this point it is proposed that the conditions in which early-years swimming takes place have the potential to build capital for the child. In the first three years parent and child spend time together in a situation where the child explores their environment and is

\footnotetext{
${ }^{1}$ The capacity of under-2s to swim may be questioned in terms of a formal definition of swimming. However, with accidental drowning being the largest cause of death in the under- 5 age group, being able to swim in a manner that aids in survival is important. While safety is a key feature in many early-years swimming programs, it has been noted that participation of this age group can enhance gross motor skill development (Zelazo \& Weiss, 2006). The focus of this paper is not on the development of swimming skills but rather on other potentials for learning that are facilitated through participation in swimming lessons for under-5s.
} 
reinforced by experts. The parent builds the overall space with which the child interacts and the swimming instructor guides them both in perception of orientation of the child's body in water and the pool. It would appear as obvious that the child embodies some forms of knowledge, skills and dispositions that become forms of capital in this extended process and they should extend them in the years between three and five. The question is how the capitals transfer into other environments, the school in particular.

Limited research has shown that early swimming can enhance some motor abilities such as balance and reaching (Sigmundsson \& Hopkins, 2010) and motor development in neonatal babies including head holding, steady sitting, and holding items (Jun, Huang, \& Dan, 2005) in able-bodied children. Other studies with children with physical disabilities have shown water activities can enhance mobility and aerobic strength (Fragala-Pinkham, Haley, \& O'Niell, 2008; Hutzler, Chacham, Bergman, \& Szeunberg, 2008). The only large study on the impact of early-years swimming conducted on young children was conducted in Germany three decades ago (Diem, 1982) when the industry was in its early stages. Since then there has been a lot of practical knowledge developed around techniques for teaching.

While the focus on swimming lessons is directed primarily at physical skills associated with swimming techniques, other aspects of the lessons can offer enhanced possibilities for learning. Such opportunities may not be unique to the swim context but, as argued earlier in this paper, the age at which children can encounter these contexts is much earlier than in other physical sports. As such, the potential to enhance learning may be made available earlier and hence offer greater possibilities than other forms of physical activities. This can now be discussed further in terms of Bourdieu's (1990) example of the exchange rates of capitals within and across economies or markets.

\section{Capital and the Exchange Economy}

In order to develop theory to understand the potential of the early-years swimming environment to shape new learning, I have adopted Bourdieu's theory of an exchange economy where cultural goods can be exchanged for other goods (Bourdieu, 1990). Within such a framework, it becomes possible to theorise the swimming environment as a site that (potentially) builds capital among young children which, in turn, can then be exchanged for other goods when they enter the formal school environment. For example, the rich mathematical language used in swimming creates new possibilities for language learning that prepares children for the mathematical discourse that they will hear in school (Jorgensen \& Grootenboer, 2011) . By incorporating this language into their habitus, young children are then able to enter school with a rich repertoire of words and interactional styles that can be exchanged for successes in school through a range of assessment practices (Bourdieu, 1977). Bourdieu's theory has been used to explain the ways in which the linguistic habitus of learners is shaped by the home environment and then either is recognised and validated in school or must undergo considerable reconstruction in order for young students to be recognised as successful learners (R. Zevenbergen, 2000). This theorisation puts the focus of analysis on the learning environment to see the ways in which context can shape learnings that may offer greater or lesser potential in the transition to school. Using the notion of 'capital', I argue that the swim environment offers potential to create opportunities for learning that prepare young children for schooling. Offering or enhancing existing forms of capital better prepares young children for schooling, and the dispositions that they acquire from participating in early-years swimming can be exchanged 
for other forms of capital within the school context. In the following sections, I discuss the potential of the early-years swimming context to add capital to under-fives.

\section{Intellectual Capital}

While intellectual capital has been colonised by the business sector to describe the accumulation of skills and dispositions that can be traded for economic wealth, it can be used to describe the general learning found in school or formal learning contexts. It is a useful construct in education to theorise the ways in which knowledge, information and experience can be built in young children so that they are predisposed to behave, act and interact in ways that position them favourably in particular contexts. The interaction between dispositions/skills/knowledge with the fields in which these can be exchanged becomes an important theorisation of how success in schools can be described. Framing learning as a form of intellect or academic knowledge, which is a type of capital, allows theorisation of dispositions that have become embodied in the learner and that will be beneficial to them in other contexts. Swimming has the potential to shape intellectual capital in forms that are valued in the context of schooling.

In the early-years swimming lessons, the teacher uses a rich language of shape and space in instructing students on how to move through the water. These terms are often richer than those found in the patterns of discourse in the home environments. Prepositions are key words in the mathematics discourse but are often limited in the home environment, particularly of disadvantaged families. Creating opportunities for exposure to these terms is critical to the learning of the complex and highly nuanced interactions that will be encountered in formal schooling. As part of the project, lesson observations have been undertaken and some of the language used in a lesson (below) highlights the instructional discourse. Observations of swim lessons show a complex and rich use of language as can be seen in the follow instructions taken from a lesson with 3 year old children:

T: big splash with two feet [holds up two fingers]

$\mathrm{T}$ : after one-two-three, we are going to push off with our hands like a rocket

$\mathrm{T}$ : eyes down in the water

$\mathrm{T}$ : we are kicking around in a circle Clinton, so kick out to me then around then back to the start

$\mathrm{T}$ : Ben arms out straight like a pencil

T: Alex, turn around so you can kick on your back, eyes up

The interactions are supporting a strong link between words and actions or concepts, thus creating opportunities for learning. In these interactions, it is possible to see how the swim environment is creating significant opportunities for developing key concepts that are foundational in mathematics learning. Not only are the words present, but the child must use body movements to link with the words, thus making for greater opportunities for deep learning. Similar opportunities in other physical activities may also be possible. The rich repertoire of words linked to actions aid in the building of knowledge and language that are valued within the school context. Acquiring these knowledges and skills can then be exchanged in the school context for some form of formal recognition, such as grades or marks or some other qualitative accreditation. As such, the knowledge acts as a form of symbolic capital, and in cases where these intellectual goods are exchanged for other goods, they become a form of institutional capital (Bourdieu, 1991).

\section{Social Capital}


While social capital has been taken up into the everyday discourses to refer to social networks, Bourdieu refers to social capital as the networks that one has and how these can be transferred to some other forms of capital. In the context of early childhood, the notion of social capital can be applied in a more rudimentary way. Socialising between children creates a sociability that is important for the transition into school where they will be expected to work with other children and adults.

Being able to mix confidently and competently with other children becomes a form of capital that has much value in educational settings. Many assessments of children in the early years of schooling are made on the basis their social skills. Being able to interact with teachers and peers is important for many school activities, so if children are confident and competent with this disposition, then they have greater chance of success in many of the activities found in formal school settings. All swim lessons are conducted in small groups and, depending on the age of the child, may have parents in the pool. There are many opportunities for the child to socially interact with other peers, thus creating opportunities for social engagement and capital building.

\section{Linguistic Capital}

At a number of levels, language becomes a form of capital that has considerable value in learning contexts. Not only are the relationships of significant importance, but also the medium through which communication occurs is important. Within the swim environment, there is potentially a rich vocabulary. Many words and phrases are used to instruct children on the activities. Teachers use terms to describe objects and locations "the red ring at the edge of the ledge" so that there are considerable adjectives and spatial language included. This rich repertoire of language positions children favourably for school. As noted in mathematics education papers (R. Zevenbergen, 2000; R Zevenbergen, 2001; R. Zevenbergen, Mousley, \& Sullivan, 2004), children from disadvantaged backgrounds often enter school without the language that prepares them for many aspects of early mathematics. The language of colour and shape are foundational to many sorting activities. Similarly, many of the spatial/preposition terms found in the swim environment are not commonly used in the home vocabulary. The swim environment provides a rich repertoire of these terms, particularly in terms of movements through the three-dimensional space. Thus, acquiring this rich language becomes a form of linguistic capital, which can then be used in the field of education where it is exchanged for institutional capital in the form of grades or other assessment practices.

The ways of interacting in the swim environment also create discursive spaces that are aligned with the patterns found in schools. Brice-Heath's (1983) (Heath, 1983) work has shown that the ways of questioning and interacting in the home can be very different from those in schools. The early childhood swim environment exposes children to the patterns of interactions commonly found in schools. The instructional discursive practices align with those found in school - e.g. "look at me", "Watch Johnny kick", "Show me how you can do ...". Early exposure to such interactional patterns is likely to prepare children for the practices that they will encounter in more formal learning settings of school.

In terms of linguistic capital, the early-years swim context has potential to enhance and consolidate not only vocabulary but also children's familiarity with the ways of interacting in the learning environment. Unlike many physical education contexts, the swim environment is highly regulated due to the safety precautions around drowning. Teachers must exert considerable control over the children to ensure their safety. In so doing, the pedagogical skills of the teachers, and their ways of interacting and controlling children, are highly 
developed. The control cannot be overt or disciplinarian in nature as the environment needs to be enjoyable to enable children to enjoy the water experience yet, simultaneously, it must ensure safety and attention. Children are thus exposed to pedagogical interactional patterns from their participation in the swim lessons.

\section{Physical Capital}

The swimming environment offers considerable potential for the development of physical capital. This is, perhaps, the most obvious capital-building aspect of the swim environment. Unlike the land environment where the space is limited to predominantly 2 dimensions, the child is able to move through a 3-dimensional space. In considering the movements needed for freestyle, such movements are not as easily made possible on land unless considerable supports are put in place. Some studies (Hutzler, et al., 2008; Malina, 2004) have shown that the flexibility of the water environment offers scope for enhancing the physical capabilities of early-years swimmers. Some of the physical skills, such as gross body movements, along with fine motor skills, may be of benefit in the school context in physical education activities.

\section{School Capital}

As noted earlier in this paper, the swim environment has greater potential than many other physical activities to build capital for young children due to the early exposure to the swim and instructional environment. While there are many aspects of learning that are foundational to the successful transition to school, the instructional discourses of the swim environment offer potential for adding capital to young children that is likely to auger well with the practices of formal schooling.

School capital refers to the skills and understandings around learning and teaching. Being able to focus on the teacher, and listen to and carry out instructions are some manifestations of school capital. The water environment is one that has a high level of safety so children are inducted into many protocols. Being in control of the learning environment is a high priority for teachers and this requires strategies for keeping children focused and on task. For example, children may be required to come to the edge of the pool, sit and wait until their lesson commences. Once in the pool, the teacher gives instructions and provides cues for different activities - such as kick, kick, kick, stop. When hearing the cue words, young children learn to comply with the instructions. These activities induct young children into the pedagogic practices that they are likely to encounter in formal learning contexts. By being exposed to these instructional protocols young children are becoming immersed in the practices that they will encounter in schools and thus this knowledge and skill set become a form of capital that can be exchanged within the school context.

\section{Exchange Economy: Transition to School}

The potential of participation in early-years swimming to enhance learning in ways that are validated in the formal school context has been theorised in this paper using Bourdieu's notion of capital. To conclude, I draw on the notion of field (Bourdieu \& in Wacquant, 1989) as this enables a theorisation of the two contexts - swimming and school - in which to locate the forms of capital. Capital gets its power only from the field within which it operates. For example, language and power are contained by the field. Speaking BBC English gains power and authority within a particular context. Such language would have little power or authority in remote communities of Central Australia or the hip-hop communities of urban settings. Thus, BBC English has strong capital only when it is located in a field 
where that language form has value. Similarly, the structuring practices of the early-years swimming context value particular skills and dispositions. The priority for the swimming context (or field) is the learning of swimming and/or water safety. Children who develop good swimming techniques are seen to have greater capital than those who do not have such well developed skills. But the focus on physical capital denies the potential of other forms of capital to be added as young children participate in this environment. Many of the attributes noted in this paper such as language or social skills have little power or value in the swim environment and hence may not be the focus of the swim schools. However, in another field, such as schooling, these dispositions have considerable value. As such, the additional learnings offered through participation in the early-years swimming environment may add more capital to young children and aid in their successful transition to school.

Participating in early-years swimming creates opportunities for young children to develop particular attributes, skills, or dispositions that will have value in another field - that is, formal schooling. By exploring a context outside formal early childhood centres, the swim environment is one site where there is considerable opportunity for young children to enhance their learning in ways that may offer considerable value in the transition to schooling. Within a Bourdieuian framework, the swimming environment creates spaces for capital building that will help in the transition to formal schooling. Children who accrue the goods offered within the swimming environment may exchange these goods - language, social skills, etc. - for other forms of capital in the school context. Taking the mathematics language noted in this paper, or the patterns of interaction, these become forms of capital in another context (or field), namely school. Participating in early-years swimming creates opportunities for young children to develop dispositions, skills, and knowledge that help in the transition to formal schooling.

\section{References}

Bourdieu, P. (1977). The economics of linguistic exchanges. Social Science Information, 16(6), 645-668.

Bourdieu, P. (1983). The forms of capital. In J. G. Richardson (Ed.), Handbook of theory and research for the sociology of education (pp. 241-258). New York: Greenwood press.

Bourdieu, P. (1990). The Logic of Practice (R. Nice, Trans.): Polity Press.

Bourdieu, P. (1991). Language and symbolic power (G. A. M. Raymond, Trans.). Cambridge: Polity Press.

Bourdieu, P., \& in Wacquant, L. (1989). Towards a reflexive sociology: A workshop with Pierre Bourdieu. Sociological Theory, 7(1), 26-63.

Department for Education Employment and Workplace Relations. (2010). The Council of Australian Governments' Early Childhood Commitment. Retrieved Dec 2, $2010, \quad$ from http://www.deewr.gov.au/Earlychildhood/Policy_Agenda/Pages/COAG.aspx

Diem, L. (1982). Early motor stimulation and personal development. A study of fourto-six-year-old German children. Journal of Physical Education, Recreation and Dance, 53, 23-25.

Fragala-Pinkham, M., Haley, S., \& O’Niell, M. (2008). Group aquatic aerobic exercise for children with disabilities. Developmental Medicine and Child Neurology, 50(11), 822-827.

Heath, S., Brice. (1983). Ways with words: Language, life and work in communities and classrooms (1989 ed.). Cambridge: University of Cambridge. 
Hutzler, Y., Chacham, A., Bergman, U., \& Szeunberg, A. (2008). Effects of movement and swimming program on vital capacity and water orientation skills of children with cerebral palsy. Developmental Medicine and Child Neurology, 40(30), 176-181.

Jorgensen, R., \& Grootenboer, P. (2011). Early years swimming as a site for early mathematical learning Proceedings of the Annual Mathematics Education Research Conference. Alice Springs: MERGA.

Jun, D., Huang, L., \& Dan, Z. (2005). The effects of infant swimming on the passage of the meconium. Journal of Nursing Science, on line.

Magnuson, K. A., Meyers, M. K., Ruhm, C. J., \& Waldfogel, J. ( 2004). Inequality in preschool education and school readiness. American Education Research Journal, 41 (1), 115-157

Malina, R. (2004). Motor development during infancy and early childhood: Overview and suggested direction for research. International Journal of Sport and Health Sciences, 2, 50-66.

Sigmundsson, H., \& Hopkins, B. (2010). Baby swimming: exploring the effects of early intervention on subsequent motor abilities. Child: Care, Health and development, 36(3), 428-430.

Webster-Stratton, C., \& Reid, M. J. (2004 ). Strengthening Social and Emotional Competence in Young Children-The Foundation for Early School Readiness and Success: Incredible Years Classroom Social Skills and Problem-Solving Curriculum. Infants \& Young Children, 17(2), 96-113.

Webster-Stratton, C., Reid, M. J., \& Stoolmiller, M. (2008). Preventing conduct problems and improving school readiness: evaluation of the Incredible Years Teacher and Child Training Programs in high-risk schools. Journal of Child Psychology and Psychiatry, 49(5), 471-488.

Zelazo, P. R., \& Weiss, M. J. (2006). Infant swimming behaviors: Cognitive control and the influence of experience. Journal of Cognition and Development 7(1), $1-25$.

Zevenbergen, R. (2000). "Cracking the Code" of Mathematics: School success as a function of linguistic, social and cultural background. In J. Boaler (Ed.), Multiple Perspectives on Mathematics Teaching and Learning. New York: JAI/Ablex.

Zevenbergen, R. (2001). Language, social class and underachievement in mathematics. In P. Gates (Ed.), Issues in teaching mathematics (pp. 38-50). London: Routledge/Falmer.

Zevenbergen, R., Mousley, J., \& Sullivan, P. (2004). Disrupting pedagogic relay in mathematics classrooms: Using open-ended Tasks with Indigenous students. International Journal of Inclusive Education., 8(4), 391-405. 